Maria Rute de Souza Araujo ${ }^{a}$

Hilton Pereira da Silva ${ }^{b}$

Ariana Kelly Leandra Silva da Silva ${ }^{c}$

\section{Avaliação situacional de biossegurança em tuberculose em Unidades Básicas de Saúde na Amazônia}

\author{
Tuberculosis biosafety measures assessment in primary health \\ care facilities from the Brazilian Amazon
}

a Universidade Estadual do Pará. Belém, PA, Brasil.

b Universidade Federal do Pará. Programa de Pós-Graduação em Saúde, Ambiente e Sociedade na Amazônia. Belém, PA, Brasil.

'Secretaria de Educação do Estado do Pará. Belém, PA, Brasil.

Contato:

Maria Rute de Souza Araujo

E-mail:

mrutearaujo@hotmail.com

Os autores declaram que a pesquisa não foi subvencionada e que não há conflitos de interesses.

O trabalho não foi apresentado em reunião científica.

Artigo baseado na dissertação de mestrado intitulada Avaliação situacional da biossegurança em tuberculose em unidade de saúde: o desafio entre o real e o ideal em saúde pública, de Maria Rute de Souza Araujo, defendida em 2011 na Universidade Federal do Pará.
Recebido: 22/09/2015

Revisado: 30/05/2016

Aprovado: 31/05/2016

\section{Resumo}

Introdução: estudo sobre a biossegurança dos profissionais de saúde que atendem pacientes com tuberculose (TB) em duas Unidades Básicas de Saúde na Amazonia brasileira, em Belém, no Pará, sendo uma um Centro de Saúde Escola e a outra uma Unidade Municipal de Saúde (UMS) que atende o maior número de casos na região. Objetivo: analisar os conhecimentos dos profissionais, a infraestrutura, as condutas e os recursos de biossegurança em TB adotados nesses serviços. Método: utilizou-se entrevista semiestruturada com quem lida com os pacientes e um roteiro de observação para avaliação da estrutura física das unidades, ambos constituídos com base nas diretrizes para TB da Sociedade Brasileira de Pneumologia e Tisiologia. Resultados: a ausência de medidas de biossegurança e a deficiência de capacitação permanente comprometem a saúde dos trabalhadores das unidades. O risco de transmissão da doença parece ser maior na UMS devido a deficiências na organização do atendimento e pela ausência de um ambiente adequado que evite o contato direto de pacientes contagiantes com as demais pessoas. Conclusão: identificou-se a necessidade urgente de mudanças no ambiente e na organização do trabalho da UMS e de um programa de capacitação permanente em TB para os profissionais de ambos os serviços.

Palavras-chave: tuberculose nosocomial; saúde do trabalhador; centro de saúde; Amazônia; saúde coletiva.

\begin{abstract}
Introduction: the research addresses the biosafety of health professionals who attend to tuberculosis (TB) patients in two health care facilities from the Brazilian Amazon - Belém, state of Pará - a school Health Center and a Primary Health Unit (UMS) where most regional TB cases are treated. Objective: to analyze the health professionals' knowledge on TB biosafety, the infrastructure, the procedures and the TB biosafety resources adopted in these services. Method: a semi-structured interview was conducted with those who deal with the patients, and an observation guide was applied to evaluate the physical structure of the areas specially designated for TB treatment, both based on the TB guidelines of the Brazilian Society of Pneumology and Tisiology. Results: the absence of biosafety measures, as well as the lack of permanent training, compromise the workers' health. The risk of TB transmission seems to be higher in the UMS due to deficiencies in care organization and to the absence of an adequate environment that could prevent the direct contact of contagious patients with other people. Conclusion: there is an urgent need for changes in the UMS's workplace and work organization, and also for a permanent TB training program for the health care professionals from both services.
\end{abstract}

Keywords: nosocomial tuberculosis; occupational safety; health center; Amazonia; public health. 


\section{Introdução}

A tuberculose (TB) é uma doença milenar, infecciosa, contagiosa, de evolução subaguda ou crônica, que ainda possui elevado índice de mortalidade no Brasil e no mundo, e está diretamente ligada à pobreza e à acelerada urbanização ${ }^{1}$. A Organização Mundial da Saúde (OMS) ainda considera a TB uma das grandes ameaças à saúde global, ainda que a taxa de mortalidade tenha caído 45\% desde 1990, a incidência esteja diminuindo em todos os continentes e parte das metas do milênio para TB tenha sido atingida na maioria dos continentes ${ }^{2}$.

Segundo dados da OMS, em 2010 foram diagnosticados e notificados 6,2 milhões de ocorrências de TB no mundo, sendo 5,4 milhões de novos casos. A Índia e a China representam $40 \%$ dos registros positivos $^{3}$.

O Brasil ocupa o $18^{\circ}$ lugar em incidência de TB dentre os 22 países que possuem $80 \%$ da carga global da doença ${ }^{4}$ e está sob monitoramento da OMS. A cada ano ocorrem 92 mil novos casos em todo o país, com taxa de incidência de 48/100 mil habitantes e prevalência de 60/100 mil habitantes. Os casos bacilíferos chegam a 49 mil por ano; os multirresistentes representam 0,9\% dos casos; e o número de óbitos pela doença é de 8,4 mil anualmente, representando uma taxa de mortalidade de 4,4/100 mil pessoas ${ }^{5}$.

No ano de 2010, o estado do Pará registrou 3.551 novos casos de todas as formas de TB. Até setembro de 2011, surgiram mais 2.437 casos no estado, com isso, o Pará ocupa o primeiro lugar do ranking da região Norte e o terceiro em incidência do país, com uma taxa de 47,8/100 mil habitantes, média de cura de $73 \%$ e abandono de tratamento em torno de $10 \%$. Os sete municípios mais acometidos e que estão em alerta são, em ordem alfabética: Abaetetuba, Ananindeua, Belém, Bragança, Castanhal, Marituba e Santarém, tendo estes prioridades no controle da doença, segundo o Ministério da Saúde e a Secretaria Estadual de Saúde ${ }^{6}$.

No Brasil, em particular na região Amazônica, ainda há escassez de dados publicados sobre biossegurança e TB nosocomial. Cotias ${ }^{7}$ define biossegurança como parte da medicina do trabalho - que trata de medidas destinadas a preservar a qualidade de vida do trabalhador - que, atualmente, tem enfoque epidemiológico, que incorpora a visão do acidente biológico.

O conhecimento acerca da TB em ambiente nosocomial pelos profissionais de saúde é fundamental diante de possível transmissão no próprio recinto laboral. Estudos no Brasil relatam elevadas prevalências e incidências entre os trabalhadores que exercem suas atividades em contato direto com pacientes com suspeita ou diagnóstico de $\mathrm{TB}^{8}$.

Pode-se prevenir a disseminação da TB em serviços de saúde, por meio de controle da infecção, obediência às diretrizes de biossegurança, capacitação e sensibilização dos profissionais sobre os riscos biológicos ${ }^{9}$.

O risco de transmissão nosocomial do Mycobacterium tuberculosis varia, principalmente, em função da prevalência local da TB e da efetividade do programa de controle da infecção nas instituições de saúde - onde pacientes com tuberculose pulmonar e laríngeas são considerados a maior fonte de infecção. Os surtos nosocomiais surgem por haver falhas no manejo, reconhecimento e isolamento adequado de pacientes com a infecção. Há necessidade da comunidade científica e dos trabalhadores da saúde se organizarem e se reconhecerem como uma população sujeita ao risco de adoecimento, e de efetivarem suas ações no sentido de minimizar os riscos potenciais nos locais onde acontecem os cuidados aos pacientes com $\mathrm{TB}^{8}$.

As Unidades Básicas de Saúde (UBS) funcionam como uma grande porta de entrada para a disseminação da TB, entretanto, na maioria das vezes, tais unidades não possuem medidas implantadas que atendam às necessidades de biossegurança, tanto para os usuários quanto para os trabalhadores, potencialmente amplificando os riscos de contágio ${ }^{10}$.

Este artigo apresenta os resultados de uma pesquisa pioneira na Amazônia, visando identificar e analisar os conhecimentos dos profissionais, os recursos infraestruturais e as condutas de biossegurança adotadas em unidades públicas de saúde, com grande fluxo de pacientes de TB, no estado do Pará.

\section{Metodologia}

O estudo foi realizado em duas instituições públicas de saúde na cidade de Belém - capital e cidade mais populosa do estado do Pará. O primeiro local investigado foi o Centro de Saúde Escola do Marco (CSE do Marco), gerenciado pela Universidade do estado do Pará - UEPA, estabelecimento que é centro de referência em pneumologia no estado. A segunda instituição foi a Unidade Municipal de Saúde do Jurunas (UMS do Jurunas). A UMS é gerenciada pela Secretaria Municipal de Saúde - SESMA. O estudo foi realizado após aprovação pelo Comitê de Ética em Pesquisa do Instituto de Ciências da Saúde da Universidade Federal do Pará (CAAE 4103.0.000.07310, parecer $\mathrm{n}^{\circ} 123 / 10$ ) e a concordância das chefias das Unidades. 
Foram adotados dois instrumentos para a coleta de dados: uma entrevista semiestruturada e um roteiro de observação para avaliação da estrutura física das unidades de saúde. Ambos instrumentos foram constituídos a partir das medidas administrativas, ambientais e de proteção respiratória recomendadas pelas III Diretrizes para TB da Sociedade Brasileira de Pneumologia e Tisiologia (SBPT) ${ }^{11}$ e pelo manual de recomendações para o controle da TB no Brasil 5 .

Primeiramente, foi aplicado o roteiro de observação - durante um período de 15 dias em cada unidade de saúde - e, posteriormente, foi feita a entrevista com os profissionais. Foram entrevistados somente os profissionais de saúde ligados efetivamente às unidades selecionadas e que atuam diretamente no Programa de Controle da Tuberculose (PCTB), nos turnos da manhã e da tarde.

Por meio do roteiro de observação, foi descrita a estrutura física das instituições no que tange à biossegurança, bem como o fluxo dos pacientes com TB e as práticas de biossegurança específicas adotadas pelos profissionais durante o atendimento. A observação foi realizada em todos os setores destinados ao PCTB, como: consultório de enfermagem, consultório médico, sala de acolhimento e sala de espera; sendo elaborado e utilizado um relatório para descrição do cotidiano das unidades de saúde. A etapa de observação foi realizada de forma não intrusiva ao cotidiano das unidades.

No CSE do Marco, participaram das entrevistas, no turno da manhã, duas enfermeiras - com tempos de atuação de 2 e 25 anos; dois técnicos de enfermagem, atuando em média há 5 meses; um médico pneumologista, que trabalha há 2 anos no PCTB; e, no turno da tarde, uma enfermeira, que desempenha sua função há 2 anos.

Quatro profissionais que atuam no PCTB participaram do estudo na UMS do Jurunas: do turno da manhã, uma médica pneumologista, atuando no programa há 4 anos; e um técnico de enfermagem, atuando há 2 meses. Do turno da tarde, participaram uma técnica de enfermagem, que atua há um ano; e uma enfermeira, que atua há 1 ano e 7 meses. Foram excluídos da pesquisa os profissionais que estavam de férias no período das entrevistas e, também, os profissionais que, esporadicamente, estavam atendendo aos pacientes com TB.

As entrevistas semiestruturadas foram divididas em duas partes, a primeira referente a dados pessoais e profissionais e a segunda com perguntas abertas, que deixavam o entrevistado livre para discorrer sobre os questionamentos a respeito de seus conhecimentos e percepções sobre as medidas administrativas, respiratórias e ambientais de biossegurança no controle de TB - não havendo tempo pré-determinado para as respostas. Todas as entrevistas foram gravadas mediante autorização dos profissionais e assinatura prévia do termo de consentimento livre e esclarecido - TCLE.

\section{Resultados}

O material coletado nas entrevistas foi analisado qualitativamente por meio da técnica de Análise de Conteúdo ${ }^{12}$. Para facilitar a compreensão, as análises foram separadas em medidas administrativas, ambientais e de proteção respiratória, que foram organizadas a partir de duas categorias: descritiva e analítica. Em seguida, foi feita a análise das percepções e conhecimentos dos profissionais relacionados às medidas de biossegurança, buscando-se estabelecer as semelhanças relevantes entre o que foi observado e o que foi relatado pelos entrevistados.

Medidas administrativas - categorias descritiva e analítica: fluxo do sintomático respiratório, agilidade no atendimento, realização de baciloscopia do escarro e aprazamento dos pacientes

No CSE do Marco, o fluxo dos pacientes com TB ocorre da seguinte forma: os sintomáticos respiratórios são encaminhados diretamente para a sala de acolhimento, onde são atendidos por técnicos de enfermagem, que solicitam duas amostras da baciloscopia de escarro - coletadas na própria instituição. O resultado fica disponível em um dia, em média, para a primeira amostra e em dois dias, para a segunda - fato comprovado por meio do livro do sintomático respiratório e de relatos das enfermeiras que atuam no programa de TB da instituição. A primeira amostra é coletada no momento do primeiro atendimento - em uma área externa da unidade, ao ar livre -, e a segunda amostra é colhida na residência do paciente. As pessoas com resultado positivo na baciloscopia, que residem na área de abrangência do CSE, são matriculadas e encaminhadas para a consulta, dando início, no mesmo dia do resultado, ao tratamento com a enfermeira. Os outros indivíduos - com baciloscopia negativa, mas com sintomas da TB - são encaminhados ao pneumologista para definição diagnóstica.

Na UMS do Jurunas não existe sala para acolhimento. Os pacientes são atendidos por enfermeiras e técnicos de enfermagem no consultório de enfermagem destinado ao atendimento de doenças transmissíveis (DT). Os sintomáticos respiratórios produzem duas amostras de escarro, sendo a primeira colhida no dia seguinte ao atendimento clínico, em jejum, e a segunda no dia posterior à primeira - ambas são realizadas na residência do paciente. Foi observado 
que, em alguns casos - como os de pacientes com sintomatologia mais evidente de TB, como tosse, emagrecimento e febre há mais de 15 dias -, os profissionais de saúde da unidade coletam a primeira amostra no dia do primeiro atendimento. Nessa instituição, não existe local destinado exclusivamente para a coleta do escarro - geralmente ela é feita dentro do banheiro, com o resultado disponibilizado em dois dias.

Casos novos, diagnosticados na própria instituição, ou em outro local, são matriculados somente no turno da tarde. Dessa forma, se o paciente comparecer no turno da manhã, este será orientado a retornar no período da tarde para ser matriculado e no dia seguinte, pela manhã, fazer a primeira consulta com o enfermeiro e iniciar o tratamento supervisionado.

Durante o período de observação dos procedimentos funcionais nas instituições, constatou-se ausência de aprazamento por horário marcado ou por dias específicos. Em ambos os locais, os pacientes, incluindo potencialmente aqueles com suspeita de TB, aguardam a consulta nos mesmos bancos longos destinados à espera dos ambulatórios médicos, juntamente com todos os outros usuários, incluindo crianças, gestantes e idosos.

No período de estudo, não foi observado nas instituições medidas para quebrar a cadeia de transmissão da TB, como busca ativa dos sintomáticos respiratórios e ações educativas voltadas para os pacientes e seus familiares.

Como visto, o CSE do Marco inicia o tratamento em 24 horas a partir da coleta da primeira baciloscopia, diminuindo o tempo de permanência (fluxo) do paciente na instituição e interrompendo a cadeia de transmissão. Por outro lado, a UMS do Jurunas leva, em média, 48 horas para iniciar o tratamento, ocasionando maior permanência do bacilífero dentro da instituição, assim como a demora na interrupção da cadeia de transmissão.

A ausência de aprazamento dos pacientes, observada nas unidades, é um fator de ampliação da transmissibilidade da doença, porém, embora preocupante, é uma norma institucional, como ilustrado pelas falas dos profissionais:

A gente trabalha com atendimento individualizado. Nós não marcamos uma hora específica pra cada paciente, a gente marca o dia e o paciente sabe que $o$ atendimento, por exemplo, vai das $7 \mathrm{~h}$ às $13 \mathrm{~h}$, no horário da manhã. Então ele tem esse período pra vir. (CSE: Profissional 2)

Na verdade, acho que teria que ter um dia só pra eles, porque quando mistura tem gente aí que tem baixa resistência, como os pacientes com diabetes e anemia [...] ou bota eles por primeiro pra gente atender e ele ir logo embora ou coloca ele em dias separados. (UMS: Profissional 2)

\section{Medidas ambientais - categorias descritiva e analítica: ventilação dos consultórios e presença de sala de espera}

No CSE do Marco são disponibilizados quatro recintos para o atendimento aos pacientes com TB: um consultório de enfermagem; uma sala onde os pacientes recebem a dose supervisionada do tratamento; um consultório médico de pneumologia; e uma sala destinada ao acolhimento dos pacientes. O consultório médico de pneumologia e de enfermagem, assim como a sala do tratamento supervisionado estão localizadas em um só corredor.

Na sala de acolhimento, os pacientes são atendidos por técnicos de enfermagem nos dois turnos. Nesta sala, existem dois tipos de ventilação, a natural - do próprio ambiente - e a artificial - com um ventilador posicionado atrás do profissional de saúde, ocasionando um fluxo de ar para fora do consultório. As janelas são do tipo pivotantes -abrem-se por completo -, e a porta do recinto permanece aberta durante todo o atendimento. As cadeiras designadas para os pacientes são fixadas no chão com parafusos, permitindo certa distância do profissional.

No consultório de enfermagem, destinado ao atendimento de TB e de outras DT, a ventilação também é natural e artificial, com janelas e portas abertas e com ventilador posicionado de modo a criar fluxo de ar entre o profissional e o paciente. No período de observação, a porta permaneceu aberta durante o atendimento, no entanto, as cadeiras dos pacientes não eram fixadas no chão. O consultório médico apresenta as mesmas características, com exceção das cadeiras dos pacientes, que são fixadas no chão.

A sala destinada ao tratamento supervisionado é localizada em uma área externa da instituição, com paredes, do tipo anteparas, a uma altura mediana, complementadas por um gradil de ferro, do tipo vergalhão, permitindo uma boa ventilação natural e entrada de sol. O local onde os pacientes aguardam para receber a medicação e o lanche é aberto, com bastante circulação de ar.

Na UMS do Jurunas são disponibilizados, para o atendimento de pacientes com TB, dois consultórios médicos e um consultório de enfermagem, onde também são realizados os acolhimentos, as consultas de enfermagem e o tratamento supervisionado. O consultório de enfermagem não possui janelas. A ventilação é artificial, realizada por meio de um ventilador de teto. No período de observação, alguns profissionais deixaram a porta fechada durante a consulta, 
enquanto outros a deixaram aberta. As cadeiras destinadas aos pacientes não estavam fixas no chão. Na sala também existe ar-condicionado, que permaneceu ligado em alguns atendimentos, tanto no período da manhã quanto da tarde. $\mathrm{O}$ atendimento médico é realizado em consultórios pequenos, sem janelas, sem ventilador de teto, com o ar-condicionado ligado e a porta fechada durante todo o atendimento.

Ambos os estabelecimentos de saúde não possuem um local específico para a espera dos pacientes com TB. Os pacientes aguardam por atendimento em corredores contíguos, junto com crianças, adultos e idosos. Nesse contexto, as instituições possuem as mesmas características, ou seja, os corredores sempre lotados de pessoas que buscam diferentes tipos de atendimento clínico, a ventilação é artificial, por meio de ventiladores direcionados aos pacientes, e os ambientes têm pouca ventilação natural.

No CSE do Marco, na sala destinada ao acolhimento dos pacientes com TB, também são atendidos os pacientes de clínicas diferentes, o que é confirmado pelo depoimento dos profissionais:

\begin{abstract}
A sala que eu trabalho deveria ter uma preocupação maior, porque eu trabalho com a psiquiatria. Os pacientes estão ali e nem sabem o que está acontecendo com eles [...] um paciente, que está com três cruzes, tá jogando bacilo no ar e daqui a um tempo a pessoa que está na mesa ao lado pode vir a ter uma infecção por tuberculose [...] eu acho que deveria ter uma sala exclusiva pra gente e não junto com outros pacientes. (CSE: Profissional 6)
\end{abstract}

Aqui os pacientes ficam misturados [...] então se fosse uma área onde a gente só atendesse esse paciente de tuberculose seria mais interessante. (CSE: profissional 1)

$\mathrm{Na}$ sala de enfermagem foi possível perceber a presença de duas enfermeiras trabalhando em mesas separadas e atendendo a pacientes com diagnósticos diferentes simultaneamente. A situação se confirma pelo depoimento que segue:

Nós temos dois profissionais no mesmo consultório. Quando o paciente não está mais bacilífero, nós atendemos dois pacientes no mesmo consultório. $O$ que não é seguro. Porque nós não atendemos somente paciente com diagnóstico estabelecido, nós atendemos o paciente que ainda está em investigação diagnóstica como o sintomático, e muitas vezes o caso é confirmado. (CSE: Profissional 2)

Na UMS do Jurunas, a falta de controle ambiental observada nos espaços assistenciais também é verbalizada pelos profissionais:

Eu acho o espaço muito pequeno e esse ventilador de teto só faz jogar o bacilo em cima da gente. (UMS: Profissional 3)
Aqui a gente não abre a janela, é todo tempo no ar-condicionado [...]. Deveria ter mais luz natural. (UMS: Profissional 2)

Medidas de proteção respiratória - categorias descritiva e analítica: máscara do tipo cirúrgica para os pacientes e máscara n95 para os profissionais de saúde

Constatou-se que os profissionais de saúde, nas duas instituições, não usam máscara durante o atendimento. No CSE do Marco, somente um paciente usava máscara enquanto permanecia na instituição - ele fazia tratamento para TB multirresistente e vinha com a máscara de sua residência. Na UMS do Jurunas não foi observado paciente com máscara cirúrgica.

O treinamento inadequado, a limitação de recursos materiais e a não sensibilização por parte dos profissionais de saúde, em ambas as instituições quanto à importância do uso de EPI para a prevenção da TB, podem ser percebidos na seguinte fala:

É recomendado, nesses treinamentos, que se use a máscara, essa é uma medida que não teve muito eco aqui na unidade, primeiro porque não tem a máscara N95 e eu acredito que esses apelos eles não sensibilizaram muito esses profissionais desse risco real [...]. Eu não me sinto confortável com a máscara, ela assusta, chama atenção, as pessoas te olham mais, olham mais para o doente quando ele está de máscara. (CSE: Profissional 3)

Neste relato fica evidente que no CSE do Marco alguns profissionais, apesar de terem o conhecimento sobre as medidas respiratórias para a prevenção da TB, não utilizam máscara para não constranger os pacientes, embora exista máscara disponível para o profissional que deseje utilizar.

\section{Percepção e conhecimento dos profissionais}

O entendimento dos profissionais de saúde sobre os riscos de contágio da TB em ambiente nosocomial e o conhecimento técnico sobre a referida doença são observações significativas que devem ser consideradas como alerta.

Ao indagar aos profissionais sobre a percepção com relação à biossegurança em TB, constatou-se que as perspectivas são voltadas difusamente à proteção individual, coletiva e à prevenção de doenças:

São diversos tipos de medidas, de diversas naturezas, para interpor barreiras entre o agente etiológico e o profissional que atende aos doentes [...]. São medidas pra proteger pessoas supostamente não infectadas, sejam profissionais ou clientes da unidade. (CSE: Profissional 3) 
Eu acho que é no sentido de prevenir a doença. (UMS: Profissional 3)

É um conjunto de coisas que a gente deveria fazer tanto para prevenir o profissional e os contatos [...] é uma coisa muito ampla. (UMS: profissional 2)

A biossegurança é você proteger tanto você quanto o doente. (CSE: profissional 5)

As principais medidas conhecidas de biossegurança em TB, apontadas nas entrevistas, limitam-se ao uso de máscara e à lavagem das mãos, como pode ser observado a seguir:

Eu conheço a máscara, tento explicar pro paciente que quando ele vai tossir deve colocar a mão ou colocar lenço ou não falar muito próximo da pessoa. (UMS: Profissional 3)

Uso de máscara, a lavagem das mãos [...]. Isso é que eu conheço. (CSE: Profissional 5)

Mesmo não havendo capacitação continuada nas instituições estudadas, percebe-se a grande importância dada por esses profissionais no tocante à necessidade de atualização própria dos procedimentos em seu local de trabalho e a busca de conhecimento, sensibilização, proteção e segurança, conforme ressaltam:

O conhecimento é a base, é o inicio do desejo de mudar alguma coisa [...]. A segunda é a sensibilização dos profissionais para esse risco muito grande de transmissão. (CSE: Profissional 3)

[A formação continuada] Serve para sensibilizar sobre a importância da pessoa em adotar as medidas de biossegurança [...]. Não adianta conhecer e não adotar as medidas. (UMS: Profissional 1)

\section{Discussão}

A biossegurança em TB tem por objetivo minimizar os riscos de se contrair a doença no ambiente de trabalho. Dessa forma, todas as medidas devem estar direcionadas, não só para quem está exposto, mas também para o meio ambiente da exposição ${ }^{13}$.

Ainda há poucos trabalhos sobre TB nosocomial em unidades de saúde. Porém, Hijjar e colaboradores, em sua pesquisa retratando a situação da TB no Brasil e no mundo, concluem que as unidades de saúde funcionam como grandes portas de entrada, tendo um número expressivo de pacientes que, antes de seu diagnóstico, circula livremente pelas dependências e que, na maioria das vezes, estas unidades não têm implantadas medidas para o diagnóstico precoce e o correto manuseio desses casos, que atendam as necessidades de isolamento e cuidados com a biossegurança ${ }^{1}$.
Diversos estudos demonstram, claramente, o risco elevado de se contrair TB entre profissionais de saúde. Em uma investigação sobre os riscos de se contrair TB entre os servidores de um hospital na cidade de Lisboa, Antunes ${ }^{14}$ calculou que o risco de contrair a doença entre os funcionários era 50\% superior à população em geral, tendo como principais causas o tempo de internação dos pacientes, o atraso no diagnóstico e no tratamento adequado de doentes infecciosos e a ventilação inadequada. Em 2011, nas unidades por nós investigadas, foram notificados 96 casos de tuberculose no CSE do Marco - destes, 46 casos bacilíferos; e na UMS do Jurunas ocorreram 64 notificações - com 41 casos bacilíferos. Embora não se tenha constatado casos entre os profissionais no período da pesquisa, esses dados demonstram que há efetivo risco de exposição ocupacional ao bacilo, requerendo medidas adequadas de biossegurança.

O Centro para o Controle e Prevenção de Doenças dos Estados Unidos (CDC), aponta que um efetivo programa de controle da TB exige identificação precoce, isolamento e tratamento das pessoas infectadas. São necessários controles que incluam: o uso de medidas administrativas, para reduzir os riscos de exposição a pessoas infectadas pelo bacilo; práticas de controle ambiental, para prevenir a disseminação e reduzir a concentração do núcleo infeccioso; e emprego de equipamentos individuais de proteção respiratória em locais onde o risco de exposição ao M. tuberculosis permaneça ${ }^{15}$.

As medidas de biossegurança em serviços de saúde adotadas no Brasil seguem as previsões da OMS e as recomendações estabelecidas nas III Diretrizes para TB da Sociedade Brasileira de Pneumologia e Tisiologia ${ }^{11}$, como também as que são citadas no Manual de recomendações para o controle da $\mathrm{TB}^{5}$. A proposta das diretrizes é revisar, de forma crítica, o que existe de mais recente na literatura científica e apresentar aos profissionais as ferramentas atuais para o enfrentamento da doença no país.

No Brasil, as medidas de controle de transmissão de TB devem levar em conta os tipos de instituição de saúde e sua função, sendo divididas em três grupos: medidas administrativas, medidas ambientais e medidas de proteção respiratória. A SBPT destacou que as medidas gerais para o ambiente ambulatorial consistem em salas de espera e de atendimento adequado e ventilado; fluxo de ar direcionado do profissional para o paciente, evitando a exposição; fluxo de pacientes com suspeita de TB separado dos outros atendimentos clínicos; pacientes com diagnósticos confirmados, com suspeita clínica sem tratamento ou com tratamento há menos de duas semanas devem usar máscara cirúrgica; e o local de coleta de escarro deve ser isolado e de preferência ao ar livre ${ }^{11,16}$. 
Na presente pesquisa, revela-se a grande diferença, entre as instituições de saúde em estudo, no tocante à agilidade em iniciar o tratamento e ao controle do fluxo desses pacientes. Na UMS do Jurunas, por exemplo, os pacientes que vão dar início à terapêutica para a TB são matriculados somente no turno da tarde. Dessa forma, se o indivíduo comparecer no turno da manhã ele poderá iniciar o tratamento apenas no dia seguinte. Já no CSE do Marco, o paciente inicia o tratamento logo após o diagnóstico, em qualquer turno. Logo, os profissionais e outros pacientes da UMS do Jurunas estão mais expostos, uma vez que é maior o tempo em que o paciente efetivamente demora para começar seu tratamento. Este fato pode ser explicado por um número reduzido de profissionais de saúde envolvidos no PCTB, principalmente no horário da manhã, no qual a procura para atendimento é maior, e pela falta de um plano de fluxo de pessoas dentro da instituição com um olhar voltado para a biossegurança e a saúde do trabalhador.

O fluxo de pacientes com TB confirmada ou suspeita, dentro dos estabelecimentos de saúde, é um dos pontos mais importantes na diminuição do risco da transmissão. Sala de espera lotada e compartilhada por muitas especialidades constitui um terreno fértil de contaminação. Falhas no reconhecimento, no manejo e no isolamento de pacientes com TB são determinantes importantes de surtos nosocomiais. É fundamental instituir um sistema de escalonamento das consultas ao longo do turno de atendimento ou mesmo um sistema de consulta com hora marcada, evitando aglomerações de pacientes potencialmente infectantes nas unidades de saúde. Filas de espera de pacientes bacilíferos no setor de radiologia, na farmácia para a recepção de medicação e no laboratório para entrega de escarro são exemplos a serem evitados na prevenção da TB nosocomial, pois os mesmos permanecem muito tempo nos setores e aumentam a chance de disseminação do bacilo ${ }^{17}$. Durante o período de observação em campo, pôde-se constatar a presença de pessoas bacilíferas esperando consultas, sentadas em bancos longos ao lado de crianças, idosos e gestantes, o que potencializa a possibilidade de contágio e desrespeita o procedimento operacional padrão de biossegurança.

Em uma pesquisa realizada entre 2002 e 2006 em um hospital universitário de Vitória, no Espírito Santo, foram identificados 25 casos notificados de profissionais de saúde com TB. Entre eles, 8 eram técnicos de enfermagem, 4 médicos, 3 enfermeiros, 2 técnicos de radiologia e 8 de outras categorias. Encontrou-se nos profissionais da área de enfermagem uma porcentagem de $44 \%$ dos casos, devido ao contato mais próximo e prolongado com os pacientes durante a jornada de trabalho ${ }^{8}$.
No Pará, verificou-se uma atuação maior da equipe de enfermagem no envolvimento do PCTB em ambas as instituições estudadas. Na UMS do Jurunas, a enfermeira faz o diagnóstico, inicia o tratamento e acompanha mensalmente todos os casos. Cabe aos profissionais médicos o atendimento dos episódios de algumas intercorrências - como reações adversas aos medicamentos -, e alta - quando em situação de cura dos sujeitos acompanhados no local. No CSE do Marco, a enfermeira tem as mesmas funções, no entanto, todos os casos de TB são acompanhados mensalmente pelos médicos e demais membros da equipe que atuam no programa.

Na observação dos aspectos arquitetônicos e de biossegurança da UMS do Jurunas, notou-se a ausência de um ambiente adequado para a circulação de pessoas, sem o contato direto com prováveis pacientes contagiantes, o que oferece um risco substancial de transmissão por via aérea entre os trabalhadores da saúde e as pessoas que frequentam a unidade, pelo grande período de permanência das pessoas sintomáticas próximas a ambientes estéreis.

Observou-se um cenário diferente no CSE do Marco, onde todos os consultórios destinados ao atendimento aos pacientes com TB apresentam janelas com ventilação natural e presença de luz solar pela manhã; os ventiladores permanecem posicionados atrás dos profissionais e as portas dos consultórios abertas durante todo o atendimento. Para a realização do tratamento diretamente observado (TDO), foi construído um anexo, em uma área externa da unidade, com bastante ventilação natural.

O potencial de transmissibilidade da doença assume maior proeminência na UMS do Jurunas pelo fato desta unidade não dispor de sistema de ventilação adequada no consultório, de local próprio para a coleta de escarro, e pela demora em iniciar o tratamento; o que não acontece no CSE do Marco, pois - apesar de ali não haver um lugar específico para a coleta -, os profissionais encaminham os seus pacientes para uma área aberta na unidade, com presença de luz solar e bastante ventilação natural, o que contribui para a diminuição de riscos de contágio. As normas preconizam que quando não for possível a coleta em local aberto esta deverá ser feita em áreas ventiladas, de forma que o procedimento não ponha em risco os profissionais e outros indivíduos que circulam na unidade de saúde ${ }^{16}$.

Durante o período de observação, constatou-se a não utilização de máscara cirúrgica pelos pacientes e máscara N95 pelos profissionais. Nosso estudo demonstra que as normas preconizadas ainda não são seguidas e que há necessidade de adoção das medidas oficialmente preconizadas pelos profissionais de saúde, principalmente na UMS do Jurunas, com 
ênfase para os funcionários que trabalham no TDO, assim como o uso de máscara cirúrgica para os pacientes sintomáticos respiratórios em ambas as unidades.

Embora haja controvérsias, segundo as normas brasileiras, as medidas de proteção respiratória consistem no uso de máscara tipo PFF2, padrão brasileiro e da União Europeia, ou N95, padrão dos Estados Unidos, pelos profissionais de saúde ou acompanhantes ao entrarem em área de alto risco de transmissão (quartos de isolamento respiratório), ambulatório para atendimento referenciado para sintomáticos respiratórios, bacilífero ou portadores de TB com suspeita de ou resistência comprovada aos fármacos antituberculose, e uso de máscara cirúrgica para pacientes com TB pulmonar ou sintomáticos respiratórios, com potencial risco de transmissão como, por exemplo, falta de estrutura de ventilação adequada na sala de espera ou no deslocamento de pacientes do isolamento para exames ou procedimentos ${ }^{16}$.

Dessa forma, Araújo ${ }^{18}$ ressalta que,

há grande necessidade das unidades de saúde em adotar programas de educação continuada sobre os aspectos epidemiológicos na transmissão da TB, os riscos ocupacionais e as medidas de biossegurança, assim como sensibilizar os profissionais nas suas ações cotidianas, pois muitos se mostraram resistentes ao uso da máscara durante o atendimento e com conhecimento insuficiente sobre as medidas de biossegurança em TB, devendo ser trabalhadas as questões do comodismo e do desinteresse pessoal de cada trabalhador, a fim de que as práticas inseguras sejam substituídas por ações que resultem em maior segurança para os trabalhadores e os pacientes por eles atendidos.

\section{Conclusões e recomendações}

Este estudo permitiu conhecer a realidade da biossegurança aplicada no controle da TB nosocomial em duas unidades de saúde amazônicas e aferir os conhecimentos sobre biossegurança dos profissionais de saúde que atuam no PCTB na região metropolitana de Belém.

Constatou-se que é reduzida a literatura existente sobre biossegurança em TB em unidades ambulatoriais, que são o primeiro local de diagnóstico e tratamento da maioria dos casos no Brasil. As publicações existentes apresentam a biossegurança, geralmente, no âmbito hospitalar, portanto, este estudo é relevante para chamar a atenção à necessidade de mais pesquisas em unidades básicas de saúde, e para analisar as medidas específicas de biossegurança existentes nas unidades, visando melhorias na saúde do trabalhador e dos pacientes.
Sob o ponto de vista da saúde do trabalhador, verificou-se nas unidades, principalmente na municipal, que a ausência das medidas de biossegurança administrativas, ambientais e respiratórias, assim como a deficiência de capacitação permanente, constituem fatores condicionantes das situações de exposição ocupacional à TB, comprometendo a qualidade dos serviços oferecidos à população e a própria segurança dos trabalhadores ${ }^{18}$.

Em relação às medidas administrativas, os fatores em comum observados nas unidades, que potencialmente contribuem para o contágio dos trabalhadores, incluem a ausência de um fluxo definido para os pacientes bacilíferos, que permita a permanência do mesmo o menor tempo possível no local; o não aprazamento por horário marcado ou por dias específicos para pacientes com TB; a falta de capacitação dos profissionais de saúde e a inexistência de uma comissão de biossegurança na instituição municipal.

Sobre os aspectos ambientais de biossegurança, as unidades de saúde estudadas possuem diferenças relevantes. O CSE do Marco comporta, em sua estrutura arquitetônica, condições favoráveis de biossegurança, atendendo às recomendações adotadas no Brasil. Por outro lado, a UMS do Jurunas apresenta em sua estrutura arquitetônica condições insalubres de trabalho.

Embora as mudanças ergonômicas em uma instituição de saúde sejam geralmente complexas e consideradas de alto custo, enfatiza-se a necessidade urgente de mudanças no ambiente de trabalho da UMS do Jurunas, com prioridade de local específico para a realização do TDO. Medidas simples como o posicionamento do ventilador, para que este produza um fluxo de ar entre o paciente e o médico, ou no sentido do médico para o paciente; a abertura de janelas que permitam mais iluminação; insolação e circulação de ar natural em consultórios e salas de espera; a não utilização de ar-condicionado ou ventilador de teto em consultórios para atendimento de TB; a organização de fluxos de pessoas, de forma a reduzir aglomerações nas salas de espera, são medidas viáveis, de baixo custo e alta eficácia sob o ponto de vista da biossegurança ${ }^{5,19}$.

É preciso também repensar os programas de treinamento, e transformar os locais de trabalho em ambientes de aprendizagem contínua, redefinindo os papéis dos trabalhadores, de forma que eles se tornem parceiros na criação de ambientes seguros e humanizados. A educação continuada deve ser um dos meios privilegiados para se processar essas transformações ${ }^{20}$.

Os resultados desta pesquisa, embora objetivamente limitados a duas unidades, servem de alerta à situação da biossegurança nos ambulatórios e centros de saúde de toda a região, pois se uma unidade que serve de escola para centenas de profissionais 
de saúde anualmente e outra de grande fluxo de TB apresentam as limitações aqui demonstradas é de se suspeitar que outras não estejam em melhor situação.

Há ainda muito a se conhecer e fazer sobre a biossegurança em TB nas unidades de saúde. Portanto, fazem-se necessários outros estudos semelhantes a este com o intuito de proporcionar um melhor conhecimento da situação, possibilitando assim a tomada de decisões informadas sobre proteção e prevenção da TB nosocomial no país.

\section{Contribuições de autoria}

Araújo MRS; Silva HP: contribuíram no delineamento, levantamento de dados, análise e interpretação dos resultados, elaboração do manuscrito e contribuição importante na sua revisão crítica e na aprovação final da versão publicada. Silva AKLS: participou da elaboração do manuscrito e contribuiu na revisão crítica e na aprovação final da versão publicada.

\section{Referências}

1. Hijjar MA, Oliveira MJPR, Teixeira GM. A tuberculose no Brasil e no mundo. Bol Pneumol Sanit. 2001;9(2):9-16.

2. World Health Organization. Global tuberculosis report 2014. Geneva: WHO; 2014.

3. American Lung Association. Trends in Tuberculosis Morbidity and Mortality [Internet]. Chicago: American Lung Association; 2013 [citado em 2016 nov 17]. Disponível em: http://www.lung. org/assets/documents/research/tb-trend-report.pdf

4. Brasil. Ministério da Saúde. Secretaria de Vigilância em Saúde. O controle da tuberculose no Brasil: avanços, inovações e desafios. Boletim Epidemiológico. 2014;44(2):1-13.

5. Brasil. Ministério da Saúde. Secretaria de Vigilância em Saúde. Departamento de Vigilância Epidemiológica. Manual de recomendações para o controle da tuberculose no Brasil. Brasília: Ministério da Saúde; 2011.

6. SESPA, 2011.

7. Cotias PMT. Procedimentos em biossegurança na tuberculose. Bol Pneumol Sanit. 2001;9(2):65-67.

8. Maciel ELN, Prado TN, Fávero JL, Moreira TR, Dietze R. Tuberculose em profissionais de saúde: um novo olhar sobre um antigo problema. J Bras Pneumol. 2009;35(1):83-90.

9. Silva FHAL, Navarro MBMA. Biossegurança e prevenção da tuberculose: a importância da qualidade do ar no interior dos serviços de saúde. Rev Patol Trop. 2013;42(2):133-146.

10. Fávero JL, Cerqueira ACB, Fregona G, Prado TN, Werner RCD, Maciel ELN. Prevalência em tuberculose em profissionais da área de enfermagem obtida por método de relacionamentos por bancos de dados, 2000 a 2008, Espírito Santo, Brasil. Rev Bras Pesqui Saúde. 2012;14(2):31-37.

11. Conde MB, Melo FAF, Marques AMC, Cardoso NC, Pinheiro VGF, Dalcin PTR et al. III Diretrizes para tuberculose da Sociedade Brasileira de
Pneumologia e Tisiologia. J Bras Pneumol. 2009;35(10):1018-1048.

12. Bardin L. Análise de conteúdo. Lisboa: Edições 70; 2002.

13. Barroso WJ. Biossegurança em tuberculose na unidade de saúde e no laboratório. Bol Pneumol Sanit. 2001;9(2):27-32.

14. Antunes AF. Epidemiologia da tuberculose: compreender para agir. In: Gomes MJM, SottoMayor R. Tratado de Pneumologia. Lisboa: Permanyer, Sociedade Portuguesa de Pneumologia; 2003. p. 625-654.

15. Centers for Disease Control and Prevention. Guidelines for Preventing the Transmission of Mycobacterium tuberculosis in Health-Care Facilities. MMWR Morb Mortal Wkly Rep. 1994;43(RR-13):1-141.

16. Brasil. Ministério da Saúde. Secretaria de Vigilância em Saúde. Departamento de Vigilância Epidemiológica. Tratamento diretamente observado (TDO) da tuberculose na atenção básica: protocolo de enfermagem. Brasília: Ministério da Saúde; 2011.

17. Gonçalves ML. Transmissão nosocomial da tuberculose: diminuindo o risco. Bol Pneumol Sanit. 2001;9(2):21-26.

18. Araújo MRS. Avaliação situacional da biossegurança em tuberculose em unidade de saúde: o desafio entre o real e o ideal em saúde pública [dissertação]. Belém: Universidade Federal do Pará; 2011.

19. Tavares DP, Gonçalves MLC, Braga PR. Recomendações para projetos de arquitetura de ambientes de tratamento de tuberculose. Rio de Janeiro: Corba; 2012.

20. Menegasso ME, Salm JF. A educação continuada e (a) capacitação gerencial: discussão de uma experiência. Revista de Ciências da Administração. 2001;3(5):27-35. 